\title{
Innovation in Nursing and Midwifery Education and Research
}

\author{
Fhumulani Mavis Mulaudzi1* \& Deborah A Chyun ${ }^{2}$ \\ ${ }^{1}$ Department of Nursing Sciences, University of Pretoria Pretoria, South Africa \\ ${ }^{2}$ New York University College of Nursing, New York, NY, USA
}

\begin{abstract}
Currently, all professions are using information communication technology (ICT) innovations that are transforming the 21 st century learning environment. Nursing and midwifery need to use ICT to provide evidence based care and acquire new skills to function efficiently in high impact technology environments. Business and community partnership will enhance an uptake and assist in mapping out programs. The purpose of this paper is to discuss innovative initiatives: blended learning, massive open online courses, simulation and embracing technology in a community oriented, primary care outreach program design. In addition, innovations in nursing research are discussed through the lens of chronic disease, the major cause of disability and death throughout the world. Innovations in nursing research are centered in three areas - study design, nursing theory and the use of technology.
\end{abstract}

Keywords: nursing education, technology, simulation, nursing research, chronic disease

\section{Introduction}

Nurses across the globe are coming together to share their experiences in education and research. This manuscript will focus on innovations in Nursing and Midwifery education and research.

\section{Innovation in Nursing and Midwifery Education}

In South Africa, currently, all professions are using Information Communication Technology (ICT) to promote innovations in teaching and learning at higher educational institutions. ICT provides a platform for flexible learning and is transforming the $21^{\text {st }}$ century learning environment. There is a need for the Nursing and Midwifery profession to use ICT and in order to provide evidence based care, and acquire new skills to function efficiently in high impact technology environments. In higher educational institutions it has become evident that the use of ICT is necessary to accommodate the $21^{\text {st }}$ century learners, who are digital citizens, requiring different pedagogical methods. These learners thrive and acquire knowledge using visual technology and words rather than the traditional methods of words alone (Critz \& Knight, 2013). Innovative initiatives described below include: blended learning, massive open online courses, simulation and embracing technology in a community oriented, primary care outreach program design.

\section{Blended Learning}

Nursing schools are now introducing innovations in teaching and learning by incorporating a blended learning environment. This blended learning intervention consists of the assimilation of classroom learning and online learning. Online learning occurs in different models. Blended learning stimulates critical thinking skills and innovative ideas among students; thus enhancing the students' learning abilities and equips the students

with innovational concepts and creative skills sets. ICT promotes discovery methods of teaching and learning which enables learners to be actively engaged in the relevant curriculum in order to build knowledge, skills, and dispositions related to the goals and objectives of the lesson (Magliaro, Lockee, and Burton, 2005). The blended learning process involves engaging learners by using stimulatory methods such as visual illustrations of the text, in a form of mind-maps, info-graphics, and sounds. At the same time discovery learning takes place, which could be in a form of experiential learning or problem based learning. This educational model moves from specific to the general and allows creativity in the student population.

*Corresponding author: Mavis.Mulaudzi@up.ac.za 
Discovery methods of instruction and learning require a conducive and nurturing environment and a paradigm shift where the lecturer is viewed as a consultant. The lecturer removes her/himself from the authority based instruction, and assumes a new role of a facilitator, thus the flipped classroom is created. Critz and Knight (2013) described a flipped classroom as a "pedagogical model" which is an innovation from the traditional classroom. They argued that the new model allows students to learn the content or subject matter on their own, outside the traditional classroom. The students will learn through innovative and new methods such as online lectures, pre-reading assign-ments and watching videos or podcasts. The classroom time is then used as a place where they can engage in debates or discussions on the issues or subject matter that they have learned. Problem solving and critical thinking skills are gained through this process.

Research has shown that there is a prominent increase of the use of cellphones worldwide. The majority of the young population in Sub-Saharan Africa own a mobile phone, making it easier for them to use personal electronic devices to learn and communicate. Access to a university now allows innovation and opens distance learning opportunities for the majority of students who were unable to access the university educational system in the past. ICT has created mechanisms to reach a large number of people making it easy for people to share resources. The use of Open Educational Resources (OER) creates access for a larger majority of the population, allowing students to be involved in educational opportunities never before accessible to this population and serves as a multiplier, enabler and is an adaptable resource.

\section{Massive Open Online Courses}

Massive Open Online Courses (MOOCs) are recent innovative additions to the online learning landscape. MOOCS are online courses that are accessible through the Website, and open to registration generally without limits on numbers or prerequisites. MOOCs are offered in many different learning modalities and generally the students register online. The course materials are free of charge and incorporate various teaching strategies during the educational process

\section{Use of Simulation}

Nurses and Midwives are expected to correlate theo- ry and practice. Innovative methods such as simulation are used to assist students to have practical skills in a safe environment without fear of hurting the patient (Ziv, Wolpe, Small, \& Glick, 2003). The use of simulation in nursing and midwifery is well accepted and adopted worldwide. Highly technically advanced manikins and simulated patients are used, giving students a safe, realistic environment to learn new skills and become proficient in their skills set through realistic practice. The students may make errors, and the simulation tools or the simulation educational staff will be able to correct and guide the student in the use of proper evidenced-based practice modalities and interventions without increased risk for the patient or stress for the student.

There are different types of simulators including standardized live patients, human patient simulators, full mission simulators, and complex and simple trainers. Training is needed for nurse educators to be able to cope with the new technology and changes happening around them; as it is critical that educators be competent in the use of simulation and incorporate this in their curriculum and educational planning. These skills may be obtained online; however institutions have an obligation to prepare their faculty. The success of simulation as an innovative mode of instruction lies with proper planning of simulated scenarios, the ability to use cost effective simulation, training of the nurse educators in the use of simulation and support from the institution ( $\mathrm{Ziv}$, Wolpe, Small \& Glick, 2003).

\section{Embracing Technology in a Community Oriented Primary Care Outreach Program}

In line with the primary health care engineering approach, a new innovation method is used to enable communities to have access to health care, using the community oriented primary care (COPC) outreach program concept. A community is divided into "wards" and the project is referred to as Ward Based OutreachTeam (WBOT) (Kinkel, De Vos \& Hugo, 2012). Wards are composed of a number of heads of households and community health care workers are assigned to those wards. The community health worker is the primary care provider for the families in these respective households. Retired nurses are used to supervise the community health workers. Students from different disciplines such as medicine, nursing, occupational therapy, and physiotherapy visit these sites for learning opportunities. The teams communicate via electronic records; personal electronic 
devices, such as mobile phones and tele-health may be utilized. The community health care workers visit families and assesse the health status of each community member. They then input the data electronically using mobile phones, and the data is captured and compiled in a web based data platform (Kinkel et al., 2012).

\section{Innovations in Research}

Innovation in research requires reflection, as the current mainstream paradigms and philosophies that form the basis of research are not viewed to be representing the African world view. Scholars assert that research done in the African continent with and about Africans must be informed of the African world view. For example, the word "Ubuntu" is a Nguni word that comes from an African proverb "Umuntu ngumuntu nga bantu" meaning a person is a person through other persons. This philosophy promotes the communal way of accomplishing things, emphasizing tenants and values such as cohesiveness, solidarity, mutual respect, sharing, participation and consensus in decision making. This philosophy requires researchers to relook at the current paradigms of research and become more inclusive of communalism. This African communal viewpoint is supported by UNESCO which states that "research institutions and practitioners are called upon to commit themselves to undertaking research that is relevant, participatory; based on indigenous culture and language of the people and that would serve the needs of the people" (UNESCO, 1996). Communalism, that is central to African ethics needs to be incorporated in the core principles in research conducted among communities in Africa whether the subjects are an individual or a group. In the case of research that is conducted between two or more countries, collaborative partnerships must involve all the different stakeholders, and ethical and cultural need may be involved in the study. Many scholars agree that health research in Africa must support ethics in the African context.

The research methods that are commensurate with the African philosophy must include participatory rural appraisal, Action Research, Emancipatory Research and mixed methods within the study designs. Participatory Action Research is broad and has different methods that can be followed such as cooperative inquiry, appreciative inquiry and other methods. These methods of research are considered ideal where the researcher aims to achieve transformation in practice through research and evidence findings.

\section{Nursing Research to Meet the Global Chal- lenges of Chronic Diseases}

It is important for nurses to understand the major health challenges faced by individuals and populations throughout the world in order to design and conduct nursing research studies that will adequately address these global problems. Chronic disease, the major cause of disability and death throughout the world, is one such problem, with cardiovascular disease (CVD) being and remaining the major cause of death. Over the next 40 years rates of heart disease and diabetes, as well as cancer and other chronic diseases including stroke, will increase, while deaths from infectious disease are expected to decrease (Abegunde, Mathers, Adam, Ortegon, \& Strong, 2007). Deaths from human immunodeficiency virus (HIV), tuberculosis (TB), and malaria will also increase worldwide. It is important to note that in developed countries, and there is every reason to believe that this will also occur in developing countries. The majority of deaths in individuals with HIV/AIDS are now related to CVD, and additionally the rates of diabetes are increasing in the population with HIV/ AIDS. In Africa, which is in the midst of the epidemiological transition from high rates of infectious diseases to high rates of chronic diseases, Africa is confronted with what the World Health Organization terms the "double burden of disease" (WHO, 2005). Nursing must focus not only on prevention and control of infectious disease, but on prevention and management of chronic diseases and their complications. It is important to note that chronic diseases and their complications can be prevented, and their progression slowed with education and support from health care providers.

When we discuss cardiovascular diseases we include: coronary heart disease (CHD), coronary artery disease (CAD), ischemic heart disease (IHD), cerebrovascular disease (CVA) or stroke, peripheral vascular disease (PVD), heart failure (HF), hypertension (HTN), rheumatic heart disease (RHD), congenital heart disease, deep vein thrombosis (DVT), and pulmonary embolism (PE). In Rwanda, HF and RHD occur frequently, and are currently important problems. However, it is likely that ischemic heart disease, stroke, and hypertension will become more commonplace unless preventive measures are instituted. CVD is and will remain the leading cause of death across the globe for the foreseeable future (WHO, 2005). 
There is a worldwide epidemic of diabetes, particularly type 2 diabetes, that is driven by overweight, and a sedentary lifestyle. Diabetes is also associated with elevated blood pressure and lipid abnormalities. Unfortunately diabetes frequently goes undiagnosed for many years, during which time complications insidiously develop such as neuropathy, retinopathy, blindness, kidney disease and CVD. Most individuals with uncontrolled diabetes will die from CVD. The ethnic minority individuals and older adults are at the highest risk of diabetes, while typically type 2 diabetes occurs in adulthood. Sadly, in westernized countries rates of diabetes are high also in children and youth, largely the result of childhood inactivity and obesity. The causes of the chronic diseases are well established, well known and similar in all regions of the world (WHO, 2005). The most important modifiable risk factors are: an unhealthy diet with excessive caloric intake, physical inactivity, and tobacco use. These risk factors, in conjunction with the non-modifiable risk factors of age and hered-ity, explain the majority of new chronic disease cases. These disease states are expressed through the development of risk factors such as increased blood pressure, elevated blood glucose levels, abnormal blood lipids (particularly elevated low density lipoprotein [LDL] cholesterol), overweight with a body mass index $[\mathrm{BMT}] \geq$ $25 \mathrm{~m} / \mathrm{kg} 2$ and obesity with a BMI $\geq 30 \mathrm{~m} / \mathrm{kg} 2$. High blood pressure and tobacco use are the strongest two risk factors most strongly associated with mortality from all causes.

The underlying determinants of chronic disease, however, the "causes of the causes", are a reflection of the major forces driving social, economic, and cultural change known as globalization, urbanization, and population aging (WHO, 2005). Nursing science is making a significant impact of decreasing levels of these risk factors and preventing chronic disease through the use of a variety of research study designs, generating nursing theory, and leading in the innovative use of technology.

\section{Nursing Research Study Designs}

Different study designs provide varying degrees of evidence, with the weakest evidence from descriptive surveys and qualitative designs, which were once the most common type of studies that nurses conducted. Nursing science has evolved with nur-ses leading quasi-experimental and experimental studies that will then serve as a basis for integrative reviews and meta-analyses, which provide the strongest levels of evidence. While interventional science is important, qualitative research is necessary as a first step in order to understand a phenomenon. The many different types of qualitative studies are important in order to obtain a complex, detailed understanding of an issue. Nursing has taken a leading role in the conduct and the dissemination of qualitative research findings.

Intervention research is critical to test the efficacy and effectiveness of our nur-sing interventions. In this type of experimental study, which we call a randomized controlled trial, we prospectively compare an intervention against a control condition, where subjects do not receive the experimental treatment. Assigning subjects to one group or the other by a random process is called randomization. This is followed by blinding the investigators or research subjects, meaning the investigators or subjects do not know whether they are receiving the experimental or control treatment, thus allowing nursing researchers to determine if the results are due to the experimental intervention. These studies do have limitations, however, as the people who participate in them are often different from the larger population, and ethical considerations must be taken into account. Nevertheless, nursing interventional science, particularly in the area of chronic disease prevention and management is strong and growing.

The problem of translating evidence from interventional studies to actual clinical practice is widely recognized, and again nursing is leading the way in bringing research from the laboratory to clinical trials, and then to the community in practical, real-world settings, as well in dissemination, implementation and outcomes evaluations in research. In addition, nursing has taken a leading role in community-based participatory research designs where community members and researchers contribute equally to the design and implementation of the study.

\section{Nursing Theory}

An important aspect of nursing interventional science is generating and testing theory. Nursing not only utilizes behavioral theories from social sciences, such as Social Cognitive Theory and the Transtheoretical Model of Behavioral Change, but is building its own body of theory, such as the Self and Family Management Framework (Grey, Schulman-Green, Knafl, \& Reynolds, 2014), and a theoretical framework for a virtual diabetes self-management as a community intervention has been utilized (Vorder- 
strasse, Shaw, Blaskovitch, \& Johnson, 2014). In a web-based platform to deliver diabetes education, which Social Cognitive Theory provided a framework including environmental, personal and behavioral components, and combined with virtual environmental constructs, built on the strengths of each, while overcoming weaknesses associated with traditional intervention methods (Vordestrasee et al., 2104). Additional theory building is occurring around the role function of community health workers, who are important sources of health information throughout the world. (Katigbak, Van Devanter, Trinh-Shevrin, Islam, \& Chyun, 2014). Key components of cultural congruence include providing social support, personal characteristics and assisting patients to interact, influencing intention and successful encouragement of adoption of healthy behaviors. Additionally, as clients shared information with their families and friends, these individuals in turn adopted healthy behaviors.

\section{Innovative Use of Technology}

Nursing is also a leader in the innovative use of technology, recognizing the benefits and limitations of technology and when it is appropriate to use technology with the identification of barriers associated with technology. A wide variety of technological approaches are available and have been successfully used in reducing chronic disease risk factors and disease itself such as mobile applications for smart phones, internet websites and social media. In one innovative diabetes self-management intervention, Second Life Impacts Diabetes Education and Self-Management (SLIDES) this avatar-based intervention attempts to overcome barriers associated with both traditional diabetes education and as asynchronous internet interventions (Vordestrasee et al., 2014). This innovative type of approach provides a large amount of quantitative and qualitative data allowing us to better understand how subjects learn and assimilate information.

\section{Conclusion}

Innovative ICT, blended learning, massive open on-line courses, simulation and use of technology in community oriented, primary care outreach programs are transforming nursing education and nursing care delivery. Research methods relevant to an African philosophy of communalism need to be employed and include participatory rural appraisal, Action Research, Emancipatory research and mixed methods approaches. Nursing is also meeting the challenge of reducing the global epidemic of chronic disease through leading the way in educational innovations, in chronic disease prevention, and behavioral change research. These include the use of a variety of study designs, theoretical innovations, and the use of technology. While globalization is making all regions of world face similar health threats, it also provides nursing with unique opportunities to be innovative and work collaboratively in terms of nursing research and educational opportunities.

\section{References}

Abegunde, D.O., Mathers, C.D., Adam, T., Ortegon, M., Strong, K. (2007). The burden and costs of chronic disease in low-income and middle-income countries. The Lancet, 370:1929-38.

Critz, C.M., Knight, D. (2013). Using the flipped classroom in graduate nursing education. Nurse Educator, 38(5):210-3.

Grey, M., Schulman-Green, D., Knafl, K., Reynolds, N.R. (2014). A revised self- and family management framework. Nursing Outlook, doi:10.1016/j.outlook.2014.10.003.

Katigbak, C., Van Devanter, N., Trinh-Shevrin, C., Islam, N., Chyun, D. Community health workers' roles in facilitating adoption of heart healthy behaviors. Circulation, 130 (suppl 2):A18996.

Kinkel, H.F., De Vos, J., \& Hugo, J. Using mobile GMS phones to capture health status assessments in COPC: Is the data plausible? Paper presented at: ICT4HEALTH Conference; 2012 Sep. 12-13; Cape Town, South Africa.

Magliaro, S.G., Lockee, B.B., \& Burton, J.K. (2005). Direct instruction revisited: A key model for instructional technology. Journal of Educational Research Technology and Development, 53, 41-55.

United Nations Educational, Scientific and Cultural Organization (UNESCO).(1996). The declarations of adult education and lifelong learning (An African position presented at the African regional consultation on adult and continiing education and the challenges on the $21^{\text {st }}$ century) UNESCO Dakar, Senegal: UNESCO.

Vorderstrasse, A.A., Shaw, R.J., Blaskovitch, J., Johnson, C. (2014). A theoretical framework for a virtual diabetes self-management community intervention. Western Journal of Nursing Research, 36:1222-37.

World Health Organization. (2005). Preventing chronic disease: a vital investment. Retrieved from http://www. who.int/chp/chronic_disease_report/en/

Ziv, A., Wolpe, P., Small, S., \& Glick, S. (2003). Simulation-based medical education: An ethical imperative. Academic Medicine, 78(8), 783-788. 